evaluations. Overall, studies were concordant on findings of high acceptability and feasibility of POC tests as well as the testing strategy used. Preference was not well demonstrated in studies. Impact was particularly well demonstrated in antenatal clinic attendees by a clustered RCT. Barriers and challenges ranged from biotechnological limitations of the tests to lack of political will. Heterogeneous methodologies employed across studies to conduct economic evaluations made it difficult to draw conclusive statements.

Conclusions Results were generally in agreement across studies, yet unsystematic methods of collecting and recording outcomes made it difficult to statistically combine outcomes. Weaknesses in the reporting of IROs limit our ability to form comprehensive contextspecific policies. Further efforts in establishing a framework for conducting implementation research is required.

\section{P1-S4.30 POINT-OF-CARE HIV TESTING WITH ORAQUICK ADVANCE HIV-1/2 ANTIBODY ASSAY: A SYSTEMATIC REVIEW OF COST OUTCOMES}

doi:10.1136/sextrans-2011-050108.174

${ }^{1} \mathrm{~B}$ Balram, ${ }^{2} \mathrm{M}$ Johri, ${ }^{2} \mathrm{D}$ Ako-Arrey, ${ }^{3} \mathrm{G}$ Lambert, ${ }^{3} \mathrm{C}$ Claessens, ${ }^{1} \mathrm{M}$ Klein, ${ }^{4} \mathrm{~J} \mathrm{M}$ Cajas, ${ }^{5}$ R Peeling, ${ }^{1} \mathrm{~N}$ Pant Pai. ${ }^{1}$ Mc Gill University, Montreal, Canada; ${ }^{2}$ Université de Montréal, Canada; ${ }^{3}$ Institut national de santé publique du Québec, Canada; ${ }^{4}$ Oueen's University, Canada; ${ }^{5}$ London School of Hygiene \& Tropical Medicine, UK

Background Recently Grading of Recommendations Assessment, Development and Evaluation (GRADE) working group called for a shift from diagnostic accuracy to emphasis on patient-centred outcomes for making policy recommendations. While meta-analyses have evaluated diagnostic accuracy of POC HIV tests, a systematic appraisal of other implementation research outcomes, such as cost, is lacking. Within this context, we reviewed global evidence on cost outcomes of OraQuick Advance HIV-1/2 Antibody Test.

Method We systematically searched six electronic databases for the period of January 1999 to January 2011. Cost outcomes with OraQuick tests were reviewed and data extracted. For economic evaluation we accepted both partial and full study designs. Outcomes were synthesised into a narrative review.

Results We identified nine studies offering economic evaluations of oral and blood based OraQuick, of which six were full economic evaluations and remaining were partial evaluations. The full economic evaluations included five Cost Effectiveness Analysis (CEA) and one Cost Utility Analysis (CUA) design; one partial evaluation was a cost comparison study and two were cost analysis studies. All studies were in the USA except one, which was from Mexico. All studies performed sensitivity analyses to explore the impact of uncertainty in their model parameters and findings. Methodological approaches applied by the authors were not standardised and program cost varied by location, but overall there was uniformity in the study conclusions. The studies concluded that OraQuick was cost effective in low prevalence settings and resulted in low rates of false positives which have favourable economic implications. The tests were found to be cost saving to the medical system, and offer the advantage of convenience in administration when compared to current standards of care. Since it was recognised that pre- and post-test counselling cost and personnel costs accounted for most of the overall costs for these rapid tests, one approach that was proposed to reduce this cost was to limit the time spent on counselling or by using lower-paid personnel for counselling activities.

Conclusion The economic evaluation results presented here can guide program managers and health policy decision makers in the research for efficient HIV testing options, for the proper allocation of healthcare resources and for adoption of models of healthcare delivery that represent the best value for money.

\section{Epidemiology poster session 4: Methodo- logical aspects: RDS \& recruitement
P1-S4.31 FAILURE OF RESPONDENT DRIVEN SAMPLING IN A TRANSGENDER POPULATION DUE TO INADEQUATE FORMATIVE WORK AND NON-NETWORK ASSOCIATED SELECTION CRITERIA

doi:10.1136/sextrans-2011-050108.175

J Risser, P Padgett, J Montealegre. University of Texas, Houston, USA

Background In 2009, the CDC funded the Transgender HIV Behavioural Surveillance pilot to assess the feasibility of using respondent driven sampling (RDS) to recruit ethnic minority male-to-female transgender (MTFTG) participants. RDS is a chain-referral strategy that restricts the number of people each participant can recruit and offers incentives for both participation and recruitment. With proper adjustment for sampling, data collected through RDS can be used to develop population prevalence estimates. RDS assumes that respondents know one another as members of the target population. Methods Because of funding constraints, formative work and data collection lasted only 4 months. Our goal was to recruit 100 MTFTG participants, at least 15 years old, who were assigned male sex at birth and who currently identified or presented as female. We established a field office in the Houston Transgender Center and identified a charismatic MTFTG interviewer. Office hours were set for early afternoon; incentives were $\$ 20$ for the interview and $\$ 10$ for each recruit (up to $\$ 50$ ). Recruiter instructions were to give the RDS coupon to known Latina or Black MTFTG.

Results Our University prohibited the hiring of our first-choice interviewer because she had a previous moral turpitude conviction. Recruitment was delayed until we hired another. We planted 11 seeds; 5 failed, 3 recruited 7 individuals in 2 waves; and 3 recruited 40 individuals with the longest chain consisting of 6 waves. We distributed 201 coupons and interviewed 48 eligible and consenting MTFTG.

Conclusions Formative work is essential to identify the size and features of the study population social networks, time and place for recruitment, and appropriate incentive. RDS requires respondents to be linked by the target attribute, in this case ethnic minority MTFTG. By restricting recruitment to transgender individuals of colour, we violated that assumption. Our sample networks were not structured around race/ethnicity; rather they were networked through work affiliations (sex worker, performer, unemployed). Recruitment also failed because the incentive was not enough to encourage performers and sex workers to participate in a health survey. Evening hours would have been better for our group's work schedules so they could participate on their way out for the evening. Our failure to get RDS initiated reinforces the importance of formative work to determine if RDS will succeed in your target population.

\section{P1-S4.32 RECRUITING VIA SOCIAL NETWORKING SITES FOR SEXUAL HEALTH RESEARCH (ASSESSING CHLAMYDIA AND HPV KNOWLEDGE)}

doi:10.1136/sextrans-2011-050108.176

${ }^{1,2,3} \mathrm{~S}$ M Garland, ${ }^{2,4} \mathrm{~J}$ D Wark, ${ }^{1,2,3} \mathrm{~S} N$ Tabrizi, ${ }^{1} Y$ Jayasinghe, ${ }^{1,3} \mathrm{E}$ Moore, ${ }^{1,3} \mathrm{~A}$ Fletcher, ${ }^{1} \mathrm{~B}$ Gunasekaran, ${ }^{1} \mathrm{~N}$ Ahmed, ${ }^{1,2} \mathrm{Y}$ Fenner. ${ }^{1}$ Department of Microbiology and Infectious Diseases, The Royal Women's Hospital, Melbourne, Australia; ${ }^{2}$ University of Melbourne, Australia; ${ }^{3}$ Murdoch Children's Research Institute, Australia; ${ }^{4}$ Department of Medicine, Royal Melbourne Hospital, Melbourne, Australia

Background Recruiting participants into population health studies has become increasingly challenging: traditional strategies have 
limitations including low participation rates and high costs. Social networking sites, commonly used for communication by young people, present an unique opportunity for innovative recruitment modalities.

Method This is part of a larger feasibility study assessing use of Facebook to recruit subjects for a novel prospective health study, the Young Female Health Initiative (YFHI). Women aged 16 to 25 years, living in Victoria, Australia were eligible to participate. An advertisement was placed on Facebook between May-and October 2010 and was visible to a random sample of eligible women. Women clicking on the advertisement were redirected to our website (http://www.yfhi.org) and invited to provide their contact details. They were contacted by a researcher and asked to complete a survey at the YFHI study site, or to complete the questionnaire online. The survey contained demographic questions, plus sensitive questions about sexual history, experience and knowledge of Chlamydia trachomatis, human papillomavirus (HPV) and HPV vaccines.

Results 551 women responded to the advertisement (recruitment could be scaled up or down by changing the advertising frequency). We enrolled 426 respondents, of whom 278 completed the survey within the time available (50\% at the study site, $50 \%$ online). Respondents' age and geographical distribution (urban, regional, rural) was representative of the target population; women over 18 years were $37 \%$ more likely to enrol and complete the survey than 16-17-year olds $(p<0.05)$. Despite the sensitive nature of some questions, over $98 \%$ of participants found the survey not at all, or only slightly, embarrassing. Overall, $63 \%$ had heard of HPV: of these, $73 \%$ knew that HPV is sexually acquired and $94 \%$ knew that it causes cancer. $78 \%$ had heard of chlamydia: those who were sexually active were more likely to know of chlamydia than virgins $(p<0.01)$, while $63 \%$ knew it could cause chronic pelvic pain, and $86 \%$ that it could cause infertility. This recruitment method also was costeffective (\$USD 20 per compliant participant).

Conclusions Results demonstrate excellent potential for such information and communication technologies (ICT) to engage young women in health research, including those from regional and rural communities, and support the use of ICT in future population-based studies, including sexual health research.

\section{P1-S4.33 EFFECTIVENESS OF RESPONDENT DRIVEN SAMPLING AMONG UNDOCUMENTED CENTRAL AMERICAN IMMIGRANT WOMEN IN HOUSTON, TEXAS, 2010}

\section{doi:10.1136/sextrans-2011-050108.177}

${ }^{1} \mathrm{~J}$ Montealegre, ${ }^{1} \mathrm{~J}$ Risser, ${ }^{1} \mathrm{~B}$ J Selwyn, ${ }^{2} \mathrm{~K}$ Sabin. ${ }^{1}$ The University of Texas School of Public Health, Houston, USA; ${ }^{2}$ WHO, Hanoi, Viet Nam

Background Respondent driven sampling (RDS) is a social research method that uses participants' social networks (SNs) to access members of hidden populations for which there is no sampling frame. RDS is widely used for HIV behavioural research among sex workers, drug users, and other hidden populations. However, it has had minimum application in migrant populations. We used RDS to conduct an HIV behavioural survey among undocumented Central American immigrant women in Houston, Texas (specifically, Guatemalan, Honduran, and El Salvadoran females, ages $18-50$ years, residing in Houston without a valid US visa or residency papers). Here we describe the effectiveness of RDS to recruit members of this population.

Methods Formative research indicated that social ties are mainly formed by country of origin, age, and number of years living in the USA. Measures of effectiveness were survey duration, participants' adoption of the recruitment system, SN density, homophily, and attainment of equilibrium. SN density is the average number of social ties per participant. Homophily is the likelihood that individuals recruit individuals like themselves; scores range from -1 to 1 , where $1=100 \%$ within-group recruitment and $0=100 \%$ random recruitment. Equilibrium is the stable sample composition that indicates independence from initial non-randomly selected participants.

Results Beginning with three initial participants, we recruited a sample of 230 immigrant women over 16 weeks. Participants adopted the recruitment system with reasonable ease (46\% recruited $\geq 1$ peers) and SNs were dense (mean SN size $=20)$. Homophily $(H)$ was moderate by country of origin (Guatemalans: $\mathrm{H}=0.52$; $\mathrm{El}$ Salvadorans: $\mathrm{H}=0.42$ ) and low by age and number of years in the USA $(\mathrm{H} \leq 0.25)$. Equilibrium was attained for all demographic and sexual behaviour characteristics.

Conclusions This study is the first to evaluate RDS in a migrant population. SNs in this population were dense, allowing recruitment to be sustained. While recruitment was moderately influenced by country of origin, women did not affiliate exclusively with those like themselves. This sociometric diversity allowed the sample to attain an equilibrium composition independent of initial participants. Overall, RDS was easy to implement, attained a large sample in a relatively short period of time, and reached an otherwise hidden population. RDS is an effective method for recruiting undocumented Central American immigrant women for HIV behavioural surveys in Houston.

\section{P1-S4.34 CAN RDS BE USED TO RECRUIT UNBIASED SAMPLES FROM THE SAME POPULATION WITH REPEATED SAMPLING?}

doi:10.1136/sextrans-2011-050108.178

J Risser, P Padgett, J Montealegre. School of Public Health, University of Texas, Houston, USA

Background Respondent Driven Sampling (RDS) is a modified snowball sampling method that allows for recruitment of a probability sample, even when we cannot enumerate the population of interest. The National HIV Behavioural Surveillance project, designed to assess trends in risky behaviours, used RDS to recruit injection drug users (IDU) and heterosexuals at high risk of HIV infection (HET). In order to examine changes in behaviours over time, the sampling method must recruit homogeneous populations, so that observed differences across cycles can be attributed to changes in behaviour, rather than to errors in recruitment. This analysis was designed to determine if crude and adjusted measures within and between the IDU and HET cycles, are homogeneous on basic demographic characteristics. Our goal to assess if the samples may be considered to be from the same target population.

Methods Data are from the Houston Texas site of NHBS-IDU 1 and 2 (2005, 2009), and HET 1 and 2 (2006, 2010). Adjusted population prevalence estimates are calculated using RDSAT, adjusting for design effect and sampling characteristics. Estimates were compared using the Mantel Haenszel test for heterogeneity.

Results Comparing IDU1 to IDU2, we found similar population estimates ( $p$ for heterogeneity $>0.05$ ) for age $40-49$ years; Black race; had current health insurance; and currently homeless. The populations differed ( $p$ for heterogeneity $<0.05$ ) by the proportion that: graduated from high school; were arrested in the last year; and visited a doctor in the last year. Comparing HET1 and HET2, we found similar population estimates for the proportions that: had health insurance; visited a doctor in the last year; were arrested in the last year; graduated from high school; and were aged 30-49. The populations differed in the percent: Black; currently homeless; and aged $18-29$.

Conclusion Using RDS to assess behaviour changes over time will be difficult if the study samples do not represent a fixed population. We 